\title{
4 \\ Are our children playing?
}

Daniela Bulgarelli and Francesca Caprino

\section{How can we be sure that children are actually playing?}

In Chapter 1, play has been defined, following Garvin, as 'a range of voluntary, intrinsically motivated activities normally associated with recreational pleasure and enjoyment'. Characteristics of play include intrinsic motivation, process oriented, enjoyment and pleasure, active engagement and internal control, and suspension of reality. Therefore, when assessing if a child is actually playing, one may ask several questions, addressing the different traits of play: are they having fun? Are they fully involved in the activity? Are they in charge of the activity? Can they choose what to do? Can they control their behaviours and the outcomes of their activities? Are they free to change and adapt their behaviours to meet their final ludic goals?

If you are used to play with children with disabilities and to observe them playing, you know that some of these questions are hard to answer. Yet, play assessment is crucial for several reasons: to help families supporting their children's right to play, to promote children's full inclusion in a wider range of play activities, to make informed decisions about educational and therapeutic programs aiming at developing and facilitating play. In line with the play definition above, this chapter addresses play assessment, and not 'play-based' assessment: in the first case, play is the focus; in the latter case, play is used as a means to evaluate other competences in children, as cognitive functioning, emotion regulation, social abilities, physical competences.

Play is a multidimensional construct and several aspects contribute to ludic activities. Thus, when adults wish to assess play, they need to identify which aspects to focus on and why, and then select the methodology of assessment. 


\section{Reasons to assess play, which aspect of play to assess and how to assess it}

In the first place, the adult has to identify the aims to be achieved through play assessment. Possible goals include:

- To assess the child's strengths and weaknesses in play;

- To assess the child's needs in play: which activities can be proposed? In which moment of the day? In which context? Which features should have the playspaces? Which toys to choose? Which play partners?

- To assess the child's preferences in play: play materials and toys, games and play activities;

- To monitor the child's progress in play.

The second step consists in choosing which is the play dimension to focus on from:

- Types of play - the adult can assess the cognitive dimension of play (practice, symbolic, constructive and rule play) or the social dimension (solitary, parallel, associative and cooperative play);

- Play developmental stages - each type of play usually emerges in a specific period of life. Practice, solitary and parallel play are typical activities of the first year; symbolic, constructive, parallel and associative play emerge at about 1824 months; children of about 3 years get involved in first forms of rule and cooperative play. As the children's competences grow, these types of play become more complex. They are also often mixed. For instance, 'hide and seek' shows features of practice, rule and cooperative play;

- Playfulness - this is the child's disposition to play. A playful child is internally motivated to play, is in control of the activities and its outcomes, is able to modulate the reality restrictions on the activity and is able to keep on going the interactions with the play partners. Therefore, playfulness partly depends on 
the child's characteristics, and partly depends on the environmental factors;

- Participation in play - full participation implies five aspects: a) the child attends and pays attention to play; b) he/she is involved in play; c) the child's preferences are addressed in the play activity; d) he/she is in the condition of exercising or improving his/her own competences in play; e) the play activity contributes to positively support or change self-confidence;

- Play contexts and environments - contextual factors refer to both space, objects, materials and to people's opinions, attitudes, knowledge, culture, etc. Different contexts (neighbourhood playgrounds, outdoor play areas, home, schoolyards, educational services, rehabilitative settings) involve different spaces, objects, materials, people, types of relationship, routines, habits that affect play in several ways.

Depending on the number and types of dimensions of play to evaluate, the assessment process will be more or less detailed.

The third step is deciding how to assess play. Different methodologies can be used:

- Interviews or questionnaires, addressed to the child or to other persons informed about his/her play activities, play preferences, etc.;

- Direct observation;

- Assessment tests. In this case, tests are usually designed to evaluate types of play or play stages.

Observation is considered the best method for assessing children's play as it provides a deeper understanding of children's behaviours in the context of everyday life and allows gathering direct information even with very young children or with children with communication impairments. Moreover, both professionals and non-professionals can assess play through observation. It is important to underline that more than one single methodology can be chosen to assess play. For example, the play observation could be preceded by a parent's interview. 


\section{Observing play}

Observing should not be confused with casual looking. Observing means carefully watching with a critical and scientific attitude to acquire new knowledge. It is a data collection process in which the human observer is the tool to measure and collect the data.

It is worth noticing that observation always includes a subjective point of view on reality, because our interpretation of situations and behaviours is guided by our implicit theories. It is well known, for example, that Eskimos have about fifty words to describe the snow: their way to conceptualize snow is different from that of European people. Thus, an Eskimo and an Italian person watching the same snow will notice and describe different things. This process affects also people who watch children playing: they will focus on different aspects according to the idea of what a child is (a sort of little adult with inadequate abilities, a person who has to be taught, a competent person to listen to; etc.) and what play is (having fun, learning, travelling to an imaginary dimension, waste of time, occupation, childish activity, etc.) For this reason, before observing play, it is crucial to bring into awareness our implicit definition of play and its several aspects: this is necessary to be able to observe and describe more precisely what we are watching. Having more than one single observer can be another useful strategy to minimize the observer's bias.

For a successful data collection through observation, one should have present what to look at. Ad hoc checklists or available play assessment tools can support the observer. Depending on the observation methodology adopted (e.g. use of an observation tool), the observer can participate to the play activity (fully or partially) or have an onlooker role. The ideal contexts to observe play are the familiar ones, such as playgrounds, home or classrooms. In those places, children usually feel comfortable and safe, because they are surrounded by relatives and friends, everyday objects and familiar routines.

The presence of severe and/or multiple disabilities can make it harder to observe and evaluate play behaviours. Thus, it is worth noticing that the effective involvement in play can be expressed through non-verbal signals that offer multiple cues to understand children's internal states, even in those with multiple disabilities: facial expressions (smiling, eyes widening, eye flashing, eye gazing), vocalizations (laughing, giggling), body movements (jumping, doing vivacious hand gestures such as hands clapping or hands waving). Even in presence of severe physical impairments, it is possible to observe enjoyment and engagement in play. The 
authentic play experience is always associated with positive emotions such as joy, excitement, curiosity, interest, satisfaction, laughter. Moreover, every child shows some kind of play ability, even at basic levels. Children unable to engage in symbolic or make believe play, or who cannot use toys in a functional way (e.g. cannot throw a ball or pile bricks), may show very simple practice play abilities such as manipulating and exploring objects. Children may not display cooperative play behaviours but be able to play alone or just close to other children without interacting with them.

\section{How to choose a tool to assess play}

To support a systematic play assessment, it is possible to choose among a wide range of tools; some of them use direct sources of information (i.e., play observation), others use indirect sources, such as interviews and questionnaires, addressed to the children or to adults who are familiar to the child.

Using a tool to support play assessment through observation can be useful for several reasons:

- The authors of the tools have precisely described which aspect of play to assess;

- If the tool is validated, i.e. if it was verified that the tool does in fact measure what it is supposed to measure, one can rely on the outcome of the assessment;

- Results of assessments in different moments in time using the same tool are comparable to each other.

Tools can be standardized or not. In the context of assessment tools, standardisation refers to the following three concepts:

- Uniformity of the administration and scoring procedures - some tools require that all children are observed in similar conditions, using the same materials, proposing the same activities, etc. Additionally, the procedures to calculate the score are also precisely explained and should be precisely followed; 
- Results are to be compared with a norm - a large number of children, divided into age groups (e.g. 5-year-olds, 6-year-olds, etc.) have been assessed using the tool and their scores were used to set the typical score of a child in a given age group. These typical scores and their standard deviations are called norms of the tool. The norms are reported in the manual or in the scientific paper describing the tool.

- Results are standardized - when using a tool to assess a competence, every child is attributed a raw score. The raw score is transformed into a standardised score to express how the child's score compares with the typical score of his/ her age group. One way of computing standardized scores is using the formula

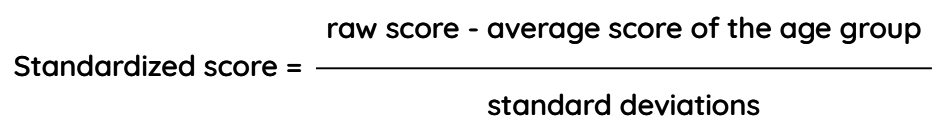

In this case, a standardized score ranging from -1 to +1 means the child's performance is typical for his/her age; a standardized score lower than -2 means the child's performance is considerably lower than what is typical for his/her age; a standardized score higher than +2 means the child's performance is considerably higher than what is typical for his/her age. Another way of computing a standardized score is through

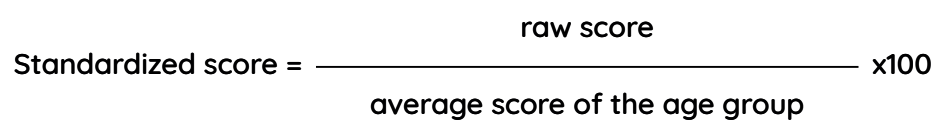

Now, a standardized score ranging between 85 and 115 means typical performance for age; standardized scores lower than 75 mean considerable lower performance for age; and standardized scores higher than 130 mean consider able higher performance for age.

Standardized tools usually were validated and thus should be preferred over non-standardized tools.

Other factors that should be considered when choosing a tool to support play assessment through observation include:

- Which aspect of play is being assessed (play preferences, play activities, playfulness, environmental context, play opportunities, etc.); 
- Who will be applying the tool - check if the tool is intended to be administered by clinicians or rehabilitation professionals (i.e., occupational therapists, speech and language therapists, psychologists, etc.), or by educators or non-professionals. Usually, this information is reported in the manual or in the scientific paper describing the tool, or it can be inferred by the professional context in which the tool has been developed;

- Tool's target group - check which is the group of people targeted by the tool (e.g. age range, country or culture of belonging, typically developing children and/or children with specific disabilities). Most of the formal assessment tools and methodologies are not specifically addressed to children with disabilities, although many of them have been tested on this target-group as well. Consequently, in some cases, the tool materials (e.g. the toys) or the testing procedures might be unsuitable for some children.

Once you have identified an assessment tool, it is possible that some adaptations of materials and/or procedures are necessary to be able to apply it to a particular group of children. Examples of adaptations include extending the time devoted to play, prompting the child, or providing physical support. Though these adaptations might be the only way of effectively applying the tool, keep in mind that they violate the standardization criteria and thus the tool's results should be used with caution.

In Chapter 11 you can find a list of available play assessment tools. Generally speaking, it is important to focus on several aspects of play: children's functioning, features of spaces and toys, characteristics of play partners, etc. The broader the view, the more informative and useful the assessment. Accordingly, it might be useful to use together two or more tools evaluating different aspects of play. For instance, the Test of Playfulness and the Test of Environmental Supportiveness were designed to be used together, the first to assess the child disposition to play and the second to assess the contextual features (e.g. spaces, materials, play partners, sensory environment) that enable or prevent play. 


\section{Assessing play: an ongoing process}

Play is a dynamic activity and children's play change over time: for these reasons, play assessment is meant to be an ongoing process. It should point out the actual competences of the child, but also look at the potential of the child. This means that play assessment should address what children can do by themselves and what they can do with a certain degree of assistance provided by an adult or by a more expert peer. This is what the soviet psychologist Vygotsky called 'the zone of proximal development', the area of learning which occurs when some kind of help is offered to the child by a person with higher competences.

Given that play assessment is an ongoing process, it is worth noticing that it can be performed in a formal or informal way. Formal assessment refers to a specific and defined time dedicated to observe play, using the methodologies that have been already described. However, informal assessment of play, by observing frequently and regularly everyday episodes and events in a more flexible way, is also necessary and useful. For example, it allows tracking the child's advances in play, possibly due to educational or rehabilitative play interventions, and it can be a starting point to decide for a formal assessment or to decide which kind of formal assessment would be preferable.

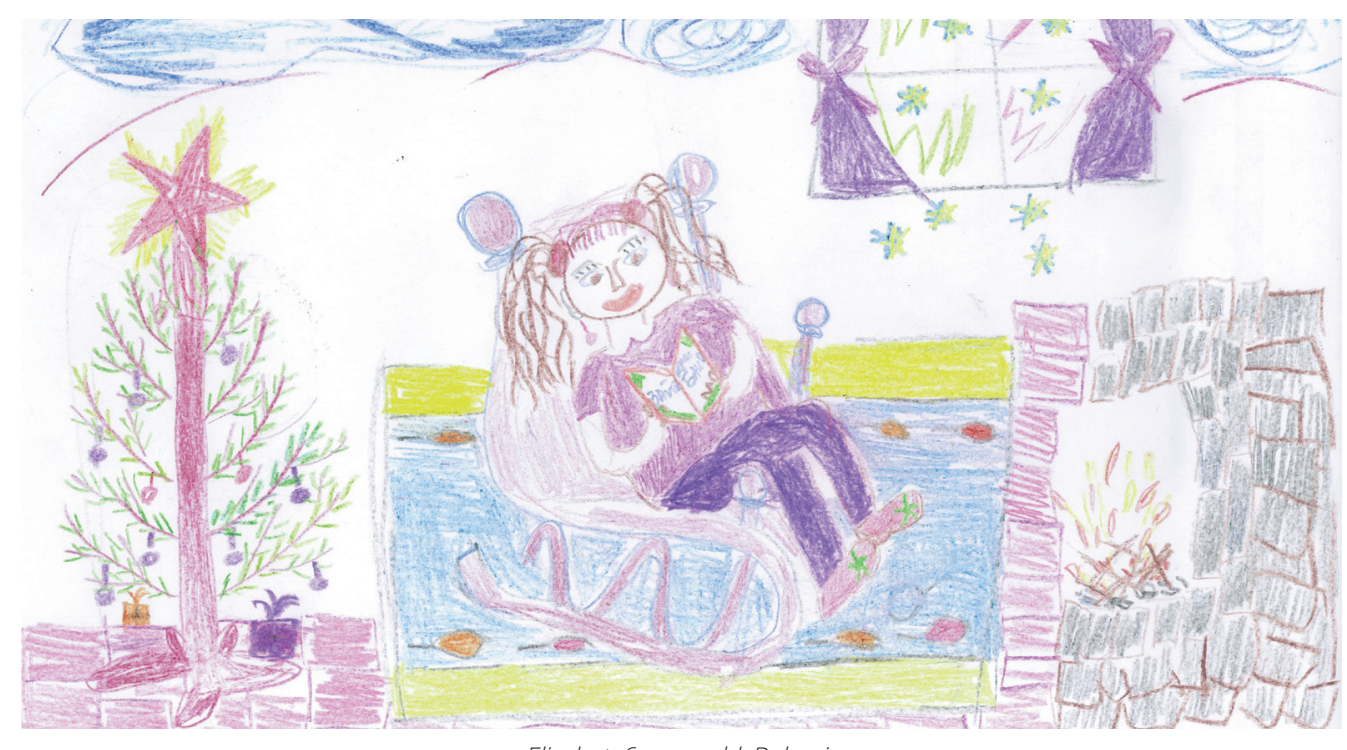

Elizabet, 6 years old, Bulgaria 


\section{Conclusion}

The assessment of play is a powerful tool for the adults who want to facilitate play for the sake of play in children with disabilities: a deeper knowledge of the child's preferences, strengths and weaknesses is the basis of any intervention aimed at supporting play.

Play assessment is not meant to measure children's abilities; rather its goal is to collect the necessary information to better create the conditions such that children can play. Most importantly, play assessment is not a goal in itself, done to label a particular child in relation to some standard, it is a tool that should be used to help children being playful and widening their play opportunities. 